\title{
Homeostasis model assessment of insulin resistance and outcome of ischemic stroke in non-diabetic patients - a prospective observational study
}

\author{
Daniel Åberg ${ }^{1,5^{*}}$ D, N. David Åberg ${ }^{1,2}$, Katarina Jood ${ }^{3}$, Lukas Holmegaard ${ }^{3}$, Petra Redfors ${ }^{3}$, Christian Blomstrand ${ }^{2,3}$, \\ Jörgen Isgaard ${ }^{1}$, Christina Jern ${ }^{3,4+}$ and Johan Svensson ${ }^{1+}$
}

\begin{abstract}
Background: Insulin resistance (IR) in relation to diabetes is a risk factor for ischemic stroke (IS), whereas less is known about non-diabetic IR and outcome after IS.

Methods: In non-diabetic IS $(n=441)$ and controls $(n=560)$ from the Sahlgrenska Academy Study on Ischemic Stroke (SAHLSIS), IR was investigated in relation to IS severity and functional outcome. IR was evaluated acutely and after 3 months using the Homeostasis model assessment of IR (HOMA-IR). Stroke severity was assessed by the National Institutes of Health Stroke Scale (NIHSS). Functional outcome was evaluated using the modified Rankin Scale (mRS) after 3 months, 2 and 7 years. Associations were evaluated by logistic regression.

Results: Higher acute and 3-month HOMA-IR was observed in IS compared to the controls (both $p<0.001$ ) and in severe compared to mild IS (both $p<0.05$ ). High acute HOMA-IR was associated with poor outcome (mRS 3-6) after 3 months and 7 years [crude Odds ratios (ORs), 95\% confidence intervals (Cls) 1.50, 1.07-2.11 and 1.59, 1.112.30, respectively], but not after 2 years. These associations lost significance after adjustment for all covariates including initial stroke severity. In the largest IS subtype (cryptogenic stroke), acute HOMA-IR was associated with poor outcome after 2 years also after adjustment for age and stroke severity (OR 2.86, 95\% Cl 1.01-8.12).

Conclusions: In non-diabetic IS patients, HOMA-IR was elevated and related to stroke severity, but after adjustment for IS severity, the associations between HOMR-IR and poor outcome lost significance. This could suggest that elevated IR mostly is a part of the acute IS morbidity. However, in the subgroup of cryptogenic stroke, the associations with poor outcome withstood correction for stroke severity.
\end{abstract}

Keywords: Insulin resistance, HOMA-IR, Ischemic stroke, Modified Rankin scale

\section{Background}

Diabetes mellitus (henceforth diabetes), type 1 as well as type 2 , is associated with increased risk for ischemic stroke (IS) [1]. Insulin resistance (IR), a hallmark of diabetes type 2 , may be present also in patients without manifest diabetes and is an independent primary risk factor for cardiovascular

\footnotetext{
* Correspondence: daniel.aberg@medic.gu.se

${ }^{\dagger}$ Christina Jern and Johan Svensson contributed equally to this work.

${ }^{1}$ Department of Internal Medicine, Institute of Medicine, the Sahlgrenska

Academy at University of Gothenburg, Gothenburg, Sweden

${ }^{5}$ Department of Internal Medicine, Sahlgrenska University Hospital, University

of Gothenburg, Blå stråket 5, SE-413 45 Göteborg, Sweden

Full list of author information is available at the end of the article
}

diseases (CVD) including IS [2, 3]. Furthermore, IR and hyperglycemia per se, regardless of a diagnosis of diabetes, are commonly seen in response to stressful situations such as critical illness and IS $[4,5]$. In a large multicenter study $(n=4537)$, diabetes was associated with worse 3-month outcome after stroke [6]. Admission hyperglycemia was associated with poor functional outcome up to one year after IS in a study population of both diabetic and non-diabetic patients [7]. A systematic review of studies in IS (diabetics included) confirmed that stress hyperglycemia was related to poor short-term outcome [5].

(c) The Author(s). 2019 Open Access This article is distributed under the terms of the Creative Commons Attribution 4.0 International License (http://creativecommons.org/licenses/by/4.0/), which permits unrestricted use, distribution, and 
In non-diabetic IS patients, hyperglycemia per se was associated with worse short-term outcome [8]. Furthermore, prediabetes tended to associate with a poor early prognosis after acute IS [9]. Also, in older non-diabetic women, homeostasis model assessment of IR (HOMAIR), but not fasting glucose or glycated hemoglobin, was an independent risk factor of coronary heart disease and stroke [10]. In recent studies of Chinese $(n=173$ and $n=1245$, respectively) $[11,12]$ and Japanese $(n=4655)$ [13] non-diabetic patients, high HOMA-IR was associated with poor outcome up to one year after the index stroke. Therefore, in summary, diabetes and/or hyperglycemia in the acute phase of IS are known risk factors for worse outcome, but relatively few long-term studies have been performed in non-diabetic patients. We analyzed IR using the HOMA-IR method [14] in non-diabetic IS patients $(n=441)$ and controls $(n=560)$ included in the Sahlgrenska Academy Study on Ischemic Stroke (SAHLSIS) $[15,16]$.

\section{Methods}

\section{Patients and controls}

The design of SAHLSIS has previously been reported [16]. Briefly, patients with first-ever or recurrent acute IS before the age of 70 years were recruited. All patients included in the present study were enrolled consecutively at four Stroke Units in western Sweden between 1998 and 2003. The controls were randomly selected either from a population-based health survey or the Swedish Population Register, to match cases regarding age $(<1$ year), sex and area of residence. Totally, there were 600 patients and 600 controls. After exclusion of all participants with diabetes, 486 patients and 560 controls were available. In the final sample, we included the 441 non-diabetic patients and 560 non-diabetic controls that had adequate blood samples for determination of HOMA-IR.

\section{Classification of stroke etiology}

Stroke etiology was classified using the Trial of Org 10172 in Acute Stroke Treatment (TOAST) criteria [17] into the subtypes large-vessel disease (LVD), small-vessel disease (SVD), cardioembolic (CE) stroke, cryptogenic stroke (when no cause was identified despite extensive evaluation) and other determined cause of stroke. In our study, we used a local protocol as specified previously $[16,18]$. According to our protocol, stroke with more than one cause was not classified as cryptogenic stroke.

\section{Stroke severity and functional outcome}

In IS patients, initial stroke severity was originally assessed by the Scandinavian Stroke Scale (SSS). The SSS is similar to the now more commonly used National Institutes of Health Stroke Scale (NIHSS) [19], with the most important exception that the scales are inverse (i.e. higher values are beneficial in SSS). We recalculated the SSS scores to NIHSS scores using the algorithm: NIHSS $=25.68-0.43 \times$ SSS [20]. We classified mild IS as NIHSS < 1.60 (SSS $=56-58$ ), moderate IS as NIHSS 1.61-5.90 ( $\mathrm{SSS}=46-55)$, and severe IS as NIHSS $>5.90$ (SSS $=1-46)$ [15]. Functional outcome was evaluated by the modified Rankin Scale (mRS) being graded 0-6, where 0 is no disability, 5 is severe disability, and 6 is dead. Evaluation of mRS was also performed after 2 and 7 years. The mRS score was dichotomized for death or dependency, i.e. poor functional outcome (mRS 3-6) versus favorable functional outcome (mRS $0-2)$.

\section{Assessment of covariates and ethical considerations}

Anthropometric parameters (body mass index, BMI) and data on hypertension, diabetes and smoking were recorded as described previously [16]. Diabetes was defined as receiving diet treatment or medication for diabetes or alternatively, as fasting plasma glucose $\geq 7.0$ $\mathrm{mmol} / \mathrm{L}$ or fasting blood glucose $\geq 6.1 \mathrm{mmol} / \mathrm{L}$ for at least two occasions [21]. Among cases, measurements performed at 3-month follow-up were used for the definition of diabetes and hypertension. Hypertension was defined by pharmacological treatment for hypertension, systolic blood pressure $\geq 160 \mathrm{mmHg}$, and/or diastolic blood pressure $\geq 90 \mathrm{mmHg}[16,22]$. BMI was calculated as $\mathrm{kg} / \mathrm{m}^{2}$. All participants or their next of kin provided written informed consent. This study was approved by the Ethics Committee of the University of Gothenburg.

\section{Biochemical analysis}

Venous blood samples were collected in the acute phase (at 1-10 days after index stroke; median 4 days), and at the 3-month follow-up in IS cases (median 101, range 85-125 days) and once in controls. Blood sampling was performed between 08:30 and 10:30 AM after overnight fasting of $>8 \mathrm{~h}$. Blood glucose or plasma glucose was analyzed, and blood glucose values were transformed to plasma glucose according to the formula: plasma glucose $=$ blood glucose $\times 1.11$. All blood and plasma concentrations of glucose and low-density lipoprotein cholesterol (hereafter LDL) were analyzed using standardized methods at the Department of Clinical Chemistry at the Sahlgrenska University Hospital. Serum levels of insulin and C-reactive protein (CRP) were analyzed by a solid-phase chemiluminescent immunometric assay on IMMULITE 2000 (Diagnostic Products Corporation, USA) using the manufacturers reagents as directed. HOMA-IR was calculated as fasting insulin (microU/L) $\mathrm{x}$ fasting glucose $(\mathrm{nmol} / \mathrm{L}) / 22.5$ [14].

\section{Statistical evaluation}

Between-group differences of means were analyzed using ANOVA followed by Tukey's HSD post hoc analysis for 
continuous variables and using chi-square tests for categorical variables. In Fig. 1, between-group differences of medians were calculated using the Kruskal-Wallis test for multiple groups followed by the Mann-Whitney Utest for pairwise comparisons.

To evaluate whether HOMA-IR was associated with functional outcome (mRS), we calculated odds ratios (ORs) and 95\% confidence intervals (CIs) using binary logistic regression analysis. In this statistical analysis, HOMA-IR was logarithmically transformed. As evaluated using the Kolmogorov-Smirnov test, HOMA-IR showed a skewed distribution prior to the logarithmical transformation, but after the logarithmical transformation, it was normally distributed. Since we intended to examine the independent effect of HOMA-IR on functional outcome, adjustments were made for predefined covariates that are related to stroke severity and well

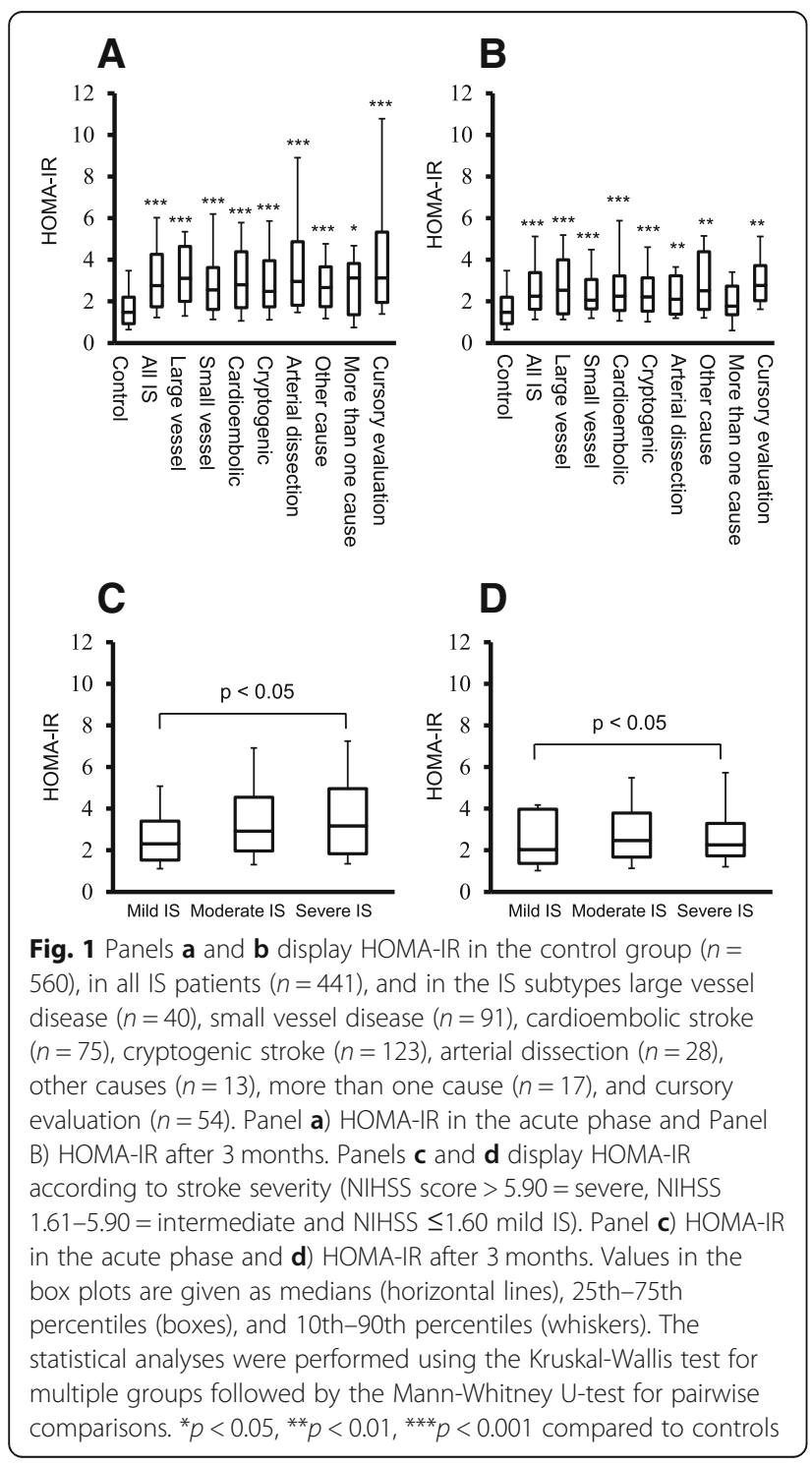

known metabolic risk factors $[15,16,23,24]$. In Table 3 , Model A included the vascular covariates age, sex, smoking, hypertension, LDL and BMI. Acute LDL was used as covariate in the model for acute HOMA-IR and 3-month LDL was used as a covariate in the model for 3-month HOMA-IR. Model B, in addition to the former covariates, also included the covariate stroke severity (NIHSS in the acute phase).

Data were analyzed using SPSS version 20.0 (SPSS Inc., Chicago, IL). $P$-values $<0.05$ were considered significant.

\section{Missing values}

In Tables 3 and 4 , out of the 441 cases at the acute phase and the 429 cases after 3 months that had blood samples available for analysis of HOMA-IR, the number in the logistic regression analysis was reduced over time due to missing values of the outcome mRS. However, for the missing values of the covariates included in the logistic regression analysis, imputed dummy variables were introduced for the categorized variables and mean values replaced the missing continuous variables.

\section{Results}

\section{Baseline characteristics}

Baseline characteristics of SAHLSIS population have been reported previously $[15,16,24,25]$. In the present study, we excluded all patients and controls having diabetes. Characteristics of the included non-diabetic participants are given in Table 1. As expected, the hypertension, smoking, history of coronary disease (= coronary event or angina pectoris) as well as atrial fibrillation were more common in patients. LDL levels and BMI did not significantly differ between patients and healthy controls, whereas acute CRP was higher in the patients. HOMA-IR was higher in the acute phase than after 3 months $(p<0.01)$, but at both time points, HOMA-IR was higher in IS patients compared to controls (both $p<0.001$; Table 1). In an additional analysis, acute HOMA-IR was found to correlate positively with $\operatorname{CRP}(p=0.014, \mathrm{r}=0.12)$.

\section{HOMA-IR, IS subtypes, and stroke severity}

HOMA-IR in controls and all IS as well as IS subtypes are given in Fig. 1. HOMA-IR both in the acute phase (Fig. 1a) and after 3 months (Fig. 1b) was significantly elevated not only in all IS, but also in all of the main IS subtypes, compared to the healthy controls.

Baseline clinical data in relation to stroke severity are presented in Table 2. Acute CRP was higher in patients with severe stroke compared to those with mild stroke. HOMA-IR was higher in severe IS than in mild IS both in the acute phase and after 3 months (both $p<0.05$; Table 2 and Fig. 1c and d). 
Table 1 Baseline characteristics and HOMA-IR in patients with ischemic stroke and controls

\begin{tabular}{|c|c|c|c|c|c|}
\hline & \multicolumn{2}{|c|}{ Cases } & \multicolumn{2}{|c|}{ Controls } & \multirow[b]{2}{*}{$p$-value } \\
\hline & $n$ & Mean \pm SEM & $n$ & Mean \pm SEM & \\
\hline Sex (ratio m/f) & 441 & $1.37 \pm 0.02$ & 560 & $1.37 \pm 0.02$ & 0.84 \\
\hline Age (years) & 441 & $56.1 \pm 0.51$ & 560 & $56.6 \pm 0.44$ & 0.44 \\
\hline Hypertension (\%) & 438 & $58 \pm 2.4$ & 559 & $36 \pm 2.0$ & $<0.001$ \\
\hline Smoking (\%) & 440 & $39 \pm 2.3$ & 560 & $18 \pm 1.6$ & $<0.001$ \\
\hline Atrial fibr (\%) & 396 & $11 \pm 1.5$ & 513 & $1 \pm 0.4$ & $<0.001$ \\
\hline CAD (\%) & 432 & $11 \pm 1.5$ & 560 & $0 \pm 0$ & $<0.001$ \\
\hline BMI $\left(\mathrm{kg} / \mathrm{m}^{2}\right)$ & 433 & $26.1 \pm 0.20$ & 559 & $26.3 \pm 0.17$ & 0.30 \\
\hline Acute LDL (mmol/L) & 395 & $3.35 \pm 0.05$ & 558 & $3.33 \pm 0.04$ & 0.75 \\
\hline Acute CRP (mg/L) & 433 & $11.4 \pm 0.98$ & 550 & $5.92 \pm 0.22$ & $<0.001$ \\
\hline Acute p-glucose & 441 & $5.69 \pm 0.06$ & 560 & $5.09 \pm 0.03$ & $<0.001$ \\
\hline 3-month glucose & 429 & $5.21 \pm 0.03$ & 560 & $5.09 \pm 0.03$ & 0.005 \\
\hline Acute insulin & 441 & $14.3 \pm 0.63$ & 560 & $8.26 \pm 0.39$ & $<0.001$ \\
\hline 3-month insulin & 429 & $12.1 \pm 0.40$ & 560 & $8.26 \pm 0.39$ & $<0.001$ \\
\hline Acute HOMA-IR & 441 & $2.84 \pm 0.18$ & 560 & $1.43 \pm 0.09$ & $<0.001$ \\
\hline 3-month HOMA-IR ${ }^{a}$ & 429 & $2.34 \pm 0.11$ & 560 & $1.43 \pm 0.09$ & $<0.001$ \\
\hline
\end{tabular}

If not otherwise stated, data are shown as means and \pm SEM. In addition, $n$ is shown as numbers vary due to missing data. Differences between cases and controls were examined by ANOVA. ns not significant, $L D L$ low-density lipoprotein, $B M I$ body mass index, CRP C-reactive protein, CAD History of Coronary Artery Disease before the event; Atrial fibr, Atrial Fibrillation before the event; Smoking, Current Smoking. ${ }^{a}$ given as geometric mean and \pm SEM

\section{HOMA-IR and functional outcome}

In the total IS population, we investigated whether HOMA-IR in the acute phase or after 3 months was associated with functional outcome. High acute HOMA-IR was associated with a poor outcome (mRS 3-6) after 3 months (crude OR 1.50, 95\% CI 1.07-2.11) and after 7 years (crude OR 1.59, 95\% CI 1.11-2.30) (Table 3). After adjusting for cardiovascular covariates in Model A, acute HOMA-IR was still associated with a poor outcome (mRS 3-6) after 3 months (OR 1.69, 95\% CI 1.16-2.46) and 7 years (OR 2.01, 95\% CI 1.31-3.10). Furthermore, deaths occurring a long time after IS may not be related to the stroke. Therefore, we performed additional analyses for the 7-year outcome in which we excluded patients who had died (= mRS 6), and these analyses showed similar associations (Table 3). However, when stroke severity was added (Model B), no significant associations remained between acute HOMA-IR and functional outcome at any time point (Table 3 ). High HOMA-IR after 3 months did not associate with poor functional outcome (mRS 3-6) after 2 or 7 years (Table 3 ).

\section{HOMA-IR and functional outcome in stroke subtypes}

Crude associations between acute HOMA-IR and outcome in the four largest subtypes of IS according to the TOAST criteria of etiology are presented in Table 4. Of note is that in the IS subtype with the largest number of

Table 2 Baseline characteristics and HOMA-IR in relation to stroke severity

\begin{tabular}{|c|c|c|c|c|c|c|c|}
\hline & \multicolumn{7}{|c|}{ Stroke severity } \\
\hline & \multicolumn{2}{|c|}{ Severe } & \multicolumn{2}{|c|}{ Moderate } & \multicolumn{3}{|l|}{ Mild } \\
\hline & $n$ & Mean \pm SEM & $n$ & Mean \pm SEM & $n$ & Mean \pm SEM & $p$-value \\
\hline Sex (ratio m/f) & 114 & $1.39 \pm 0.05$ & 167 & $1.37 \pm 0.04$ & 160 & $1.36 \pm 0.04$ & 0.59 \\
\hline Age (years) & 114 & $56.1 \pm 0.95$ & 167 & $57.0 \pm 0.80$ & 160 & $55.2 \pm 0.89$ & 0.49 \\
\hline Hypertension (\%) & 112 & $57 \pm 4.7$ & 166 & $64 \pm 3.7$ & 160 & $52 \pm 4.0$ & 0.39 \\
\hline Smoking (\%) & 113 & $48 \pm 4.7$ & 167 & $30 \pm 3.6$ & 160 & $42 \pm 3.9$ & 0.34 \\
\hline Atrial fibr (\%) & 113 & $14 \pm 3.6$ & 149 & $11 \pm 2.5$ & 150 & $8 \pm 2.2$ & 0.11 \\
\hline CAD (\%) & 97 & $16 \pm 3.5$ & 167 & $10 \pm 2.4$ & 158 & $9 \pm 2.3$ & 0.071 \\
\hline BMI $\left(\mathrm{kg} / \mathrm{m}^{2}\right)$ & 110 & $25.6 \pm 0.42$ & 166 & $26.7 \pm 0.36$ & 157 & $25.7 \pm 0.29$ & 0.86 \\
\hline Acute LDL (mmol/L) & 98 & $3.22 \pm 0.10$ & 147 & $3.40 \pm 0.08$ & 150 & $3.39 \pm 0.08$ & 0.18 \\
\hline Acute CRP (mg/L) & 113 & $16.4 \pm 2.32$ & 164 & $10.7 \pm 1.77$ & 156 & $8.65 \pm 0.97$ & 0.002 \\
\hline Acute p-glucose & 114 & $6.14 \pm 0.15$ & 167 & $5.64 \pm 0.09$ & 160 & $5.41 \pm 0.04$ & $<0.001$ \\
\hline 3-month p-glucose & 106 & $5.21 \pm 0.05$ & 171 & $5.23 \pm 0.05$ & 152 & $5.17 \pm 0.05$ & 0.69 \\
\hline Acute insulin & 114 & $15.2 \pm 1.28$ & 167 & $15.1 \pm 1.10$ & 160 & $12.9 \pm 1.05$ & 0.18 \\
\hline 3-month insulin & 106 & $12.5 \pm 0.88$ & 171 & $13.4 \pm 0.81$ & 152 & $10.5 \pm 0.48$ & 0.03 \\
\hline Acute $H O M A-\mathbb{R}^{a}$ & 114 & $3.22 \pm 0.41$ & 167 & $2.95 \pm 0.29$ & 160 & $2.47 \pm 0.28$ & 0.02 \\
\hline 3 month HOMA-IR & 106 & $2.48 \pm 0.24$ & 171 & $2.50 \pm 0.21$ & 152 & $2.00 \pm 0.13$ & 0.02 \\
\hline
\end{tabular}

The patients were categorized using the acute National Institutes of Health Stroke Scale (NIHSS) as having severe (NIHSS score 5.91-25.25), moderate (NIHSS score 1.61-5.90) or mild (NIHSS stroke $0-1.60$ ) stroke. If not otherwise stated, data are shown as means (if not otherwise stated) and \pm SEM. In addition, $\mathrm{n}$ is shown as numbers vary due to missing data. Differences between severe and mild stroke were examined by ANOVA. ns not significant, $L D L$ low-density lipoprotein, BMI body mass index, CRP C-reactive protein, CAD History of Coronary Artery Disease before the event; Atrial fibr, Atrial Fibrillation before the event; Smoking, Current Smoking. ${ }^{a}$ given as geometric mean and \pm SEM 
Table 3 Risk of poor IS outcome per unit increase in log HOMA-IR

\begin{tabular}{|c|c|c|c|c|}
\hline & $n$ & Crude & Model A & Model B \\
\hline \multicolumn{5}{|l|}{ Acute HOMA-IR } \\
\hline 3 months & 423 & $1.50(1.07-2.11)^{*}$ & $1.69(1.16-2.46)^{* *}$ & $1.12(0.60-2-06)$ \\
\hline 2 years & 436 & $1.22(0.86-1.71)$ & $1.32(0.90-1.92)$ & $0.89(0.51-1.55)$ \\
\hline 7 years Deaths (mRS 6) included & 294 & $1.59(1.11-2.30)^{*}$ & $2.01(1.31-3.10)^{* *}$ & $1.75(0.97-3.16)^{t}$ \\
\hline 7 years Deaths (mRS 6) excluded & 254 & $1.50(0.96-2.33)^{\dagger \dagger}$ & $1.72(1.03-2.87)^{*}$ & $1.23(0.59-2.57)$ \\
\hline \multicolumn{5}{|l|}{3 months HOMA-IR } \\
\hline 3 months & 420 & $1.25(0.84-1.85)$ & $1.53(0.95-2.45)^{\ddagger}$ & $1.27(0.69-2.42)$ \\
\hline 2 years & 426 & $1.15(0.77-1.71)$ & $1.24(0.77-1.98)$ & $1.04(0.56-1.94)$ \\
\hline 7 years Deaths (mRS 6) included & 289 & $0.97(0.64-1.48)$ & $1.03(0.61-1.74)$ & $0.91(0.49-1.70)$ \\
\hline 7 years Deaths (mRS 6) excluded & 257 & $0.93(0.56-1.55)$ & $0.80(0.43-1.50)$ & $0.70(0.31-1.58)$ \\
\hline
\end{tabular}

subjects, cryptogenic stroke, high acute HOMA-IR was more robustly associated with poor functional outcome after 3 months (crude OR 3.65, 95\% CI $1.53-8.72 ; p<0.01$ ) and 2 years (crude OR 4.77, 95\% CI $1.93-11.8 ; p<0.001)$. This contrasted to lower, non-significant ORs in the other subtypes. Due to the

Table 4 Risk of poor outcome in IS subtypes per unit increase in acute log HOMA-IR

\begin{tabular}{lll}
\hline & $\mathrm{n}$ & Crude OR \\
\hline 3 months & 37 & $0.73(0.19-2.85)$ \\
Large vessel disease & 85 & $0.31(0.07-1.30)$ \\
Small vessel disease & 74 & $1.81(0.85-3.85)$ \\
Cardioembolic stroke & 119 & $3.65(1.53-8.72)^{* *}$ \\
Cryptogenic stroke & & \\
2 years & 40 & $0.39(0.10-1.47)$ \\
Large vessel disease & 90 & $0.37(0.11-1.20)$ \\
Small vessel disease & 74 & $1.29(0.61-2.73)$ \\
Cardioembolic stroke & 122 & $4.77(1.93-11.8)^{* * *}$ \\
Cryptogenic stroke & & \\
7years Deaths (mRS 6) included & 30 & $2.36(0.59-9.45)$ \\
Large vessel disease & 61 & $0.92(0.30-2.77)$ \\
Small vessel disease & 51 & $1.55(0.70-3.42)$ \\
Cardioembolic stroke & 93 & $2.09(0.90-4.87)^{\dagger}$ \\
Cryptogenic stroke & & \\
7years Deaths (mRS 6) excluded & & $0.76(0.14-4.03)$ \\
Large vessel disease & 22 & $1.16(0.36-3.77)$ \\
Small vessel disease & 59 & $1.25(0.42-3.77)$ \\
Cardioembolic stroke & 35 & $2.30(0.74-7.16)$ \\
Cryptogenic stroke & 84 & \\
\hline Odds ratios (ORs) and 95\% confden & &
\end{tabular}

Odds ratios (ORs) and $95 \%$ confidence intervals (Cls) of having a poor outcome ( $m R S \geq 3$ ) for the main etiologies of IS (according to TOAST) at different time points per unit increase in acute log-HOMA-IR. ${ }^{\dagger} p=0.08$, ${ }^{* *} p<0.01,{ }^{* * *} p<0.001$ low number of patients in each IS subtype, we found it proper to adjust for covariates only in this largest subtype, i.e. cryptogenic stroke (numbers with poor outcome: $n=$ 19 at 3 months, $n=19$ at 2 years, $n=17$ at 7 years and $n=$ 8 at 7 years excluding deaths), and to restrict the number of covariates to two. After adjustment for the two covariates with the largest influence on outcome (age and acute NIHSS), the association between acute HOMA-IR and 3month outcome lost significance (adjusted OR 1.66, 95\% CI 0.50-5.44, data not shown), whereas the association with 2-year outcome remained (adjusted OR 2.89, 95\% CI $1.02-8.20 ; p=0.047$, data not shown). There was no statistically significant association between acute HOMA-IR and 7-year outcome, either with or without exclusion of mRS 6, in any IS subtype (Table 4).

HOMA-IR after 3 months did not associate with outcome after 2 or 7 years in any IS subtype (data not shown).

\section{Discussion}

In this study of well-characterized non-diabetic IS patients, we found that HOMA-IR declined between the acute phase and the 3-month follow-up. However, at both time points, HOMA-IR was higher in IS patients than in the population-based non-diabetic controls, and this was true for all main etiological subtypes of IS. Furthermore, HOMA-IR was higher in severe than in mild IS at both time points. Acute HOMA-IR was associated with poor functional outcome (mRS 3-6) after 3 months and 7 years, but these associations abided after adjustment for stroke severity. However, in cryptogenic stroke (the most common IS subtype), the association between high acute HOMA-IR and poor outcome after 2 years was independent of age and stroke severity. In contrast, HOMA-IR after 3 months did not associate with functional outcome at any time point of follow-up.

To the best of our knowledge, this is the first study to investigate HOMA-IR in relation to both stroke severity 
and long-term outcome in a large cohort of non-diabetic Caucasian patients with IS. We found that both acute and 3-month HOMA-IR were higher in severe compared to mild stroke. Although hyperglycemia has been found to be common after IS $[1,5,26]$, the association between IR and stroke severity in non-diabetic patients has been less investigated and with different findings. In the study by Calleja et al., in which insulin treatment was an exclusion criteria, NIHSS score did not differ between HOMA-IR tertiles $(n=109)$ [27], while Bas et al. found that non-diabetic IS patients with admission HOMA-IR $>2.7$ had marginally increased NIHSS score compared to those with HOMA-IR $<2.7 \quad(n=180)$ [28]. The present relatively large study $(n=441)$ therefore establishes that IR is related to stroke severity also in nondiabetic IS patients.

In the present study, high acute HOMA-IR was associated with poor functional outcome 3 months and 7 years after the index stroke. These association between high acute HOMA-IR and poor functional outcome remained after adjustment for cerebrovascular risk factors (Model A), but did not withstand adjustment for both vascular confounding factors and initial stroke severity (Model B). Thus, when assuming IR is not a mediator of ischemic injury, but rather secondary to the injury and thereby adding initial stroke severity as a covariate, the associations disappeared. In previous studies, poor functional outcome up to one year after IS was observed in patients with hyperglycemia and/or diabetes $[6-8,29,30]$. Furthermore, hyperglycemia was related to increased short- (30 days) and long-term ( 1 and 6 year) mortality in an IS population including diabetic patients [31]. Baird et al. [32] found that prolonged poststroke hyperglycemia was independently associated to poor short-term clinical outcome as well as infarct expansion. Possibly, the effects of persistent hyperglycemia in the penumbra of IS might be detrimental, resulting in reduced penumbral salvage, larger final infarct size, and worse clinical outcome [33]. In Chinese ( $n=173$; non-diabetics) [11] and Japanese $(n=4655$; no insulin treatment) [13] patients, high HOMA-IR index was associated with poor short-term functional outcome (mRS at discharge and 3 months, respectively). Finally, in a Chinese non-diabetic IS cohort $(n=1245)$ followed up to one year after IS, HOMA-IR in the acute phase was associated with increases in the risks of death, stroke recurrence and poor outcome (mRS 3-6), but not with dependence (mRS 3-5) [12]. However, there is no previous long-term study in a non-diabetic Caucasian IS population, and the importance of HOMA-IR could be different in Asian and Caucasian patients [34, 35].

There are a few studies that might implicate different mechanisms of how IR is unfavorable after IS. Two smaller studies $(n=109$ and $n=180)$ specifically investigated the association between HOMA-IR and 3-month functional outcome in non-diabetic IS patients that had received intravenous thrombolysis, and an independent association between high HOMA-IR and poor outcome was observed $[27,28]$. In a large register study of IS patients treated with intravenous thrombolysis $(n=16049)$, high admission blood glucose was associated with an increased risk of symptomatic intracerebral hemorrhage, higher mortality, and poor functional outcome as assessed by mRS after 3 months [36]. During the inclusion period for the present study, very few patients received thrombolytic treatment in our region, and among the patients studied here only 3 patients received thrombolysis. Therefore, intracerebral hemorrhage due to thrombolysis is not a relevant mechanism to explain our association between HOMA-IR and long-term outcome of IS.

Acute illness, including IS, is associated with stress hyperglycemia and increased IR [4, 5, 37]. In line with this, we observed that HOMA-IR was higher in the acute phase of IS than after 3 months. In addition, we found a positive correlation between HOMA-IR in the acute phase and acute CRP. Possibly, acutely elevated IR can be seen as part of the acute IS morbidity whereas IR after 3 months more reflects IR under steady state conditions. In contrast to acute HOMA-IR, no associations to outcome were observed for 3-month HOMA-IR. It may be speculated that as most neurological recovery occurs early after the ischemic event, IR in the acute phase has a larger impact on stroke recovery and thus on outcome than IR 3 months after the event. On the other hand, IR is a principle component of the metabolic syndrome along with central obesity, hypertension, and dyslipidemia, which contributes to bad outcome in many different contexts [38, 39]. Therefore, behavioral, lifestyle and/or therapeutic interventions that improve glucose homeostasis could also reduce metabolic derangements and potentially improve IS outcome. In the IRIS trial, which included non-diabetic patients who had insulin resistance and a recent history of IS or transient ischemic attack (TIA), the risk of stroke or myocardial infarction was lower in patients receiving pioglitazone treatment [40].

We found that HOMA-IR was elevated both in the acute phase and after three months in all main IS subtypes, i.e. large vessel disease, small vessel disease, cardioembolic stroke and cryptogenic stroke compared to the controls, suggesting that IR is a common feature of IS regardless of IS subtype. In the largest subtype that represents about one quarter of IS cases in the age group under study here, i.e. cryptogenic stroke, we observed a significant association between high acute HOMA-IR and poor outcome after 3 months and 2 years. Importantly, this association remained after adjustment for age and stroke severity. By definition, there 
is no obvious cause of IS in cryptogenic stroke [41], but infections/inflammation and hypercoagulability have been proposed as underlying mechanisms [42]. Although highly speculative, in the absence of obvious underlying mechanisms such as atherosclerosis or atrial fibrillation in cryptogenic stroke, the relative role of acute IR for outcome could be more pronounced. However, the association between acute HOMA-IR and 2-year outcome in cryptogenic stroke must be considered as a preliminary observation until confirmed in future studies having this as a primary hypothesis.

\section{Strengths and limitations}

Our study included a relatively large sample of consecutive and well-characterized IS patients and populationbased controls, and few patients were lost to follow-up. Only Caucasian participants were studied, which might limit the generalizability of our results, but could be of importance $[34,35]$ when comparing our results with those of studies with Asian origin [11-13]. Our patients were classified into etiological subtypes of IS, but despite the relatively large study sample, we did not have enough power to adjust for stroke severity and other possible confounders of outcome in all subtypes. Furthermore, the relatively young mean age of the participants (56 years) could have favored the inclusion of less severe IS cases with low fatality, and to some degree disfavored the inclusion of older patients with IS due to cardiovascular causes. We estimated IR using the HOMA-IR, which is commonly used in clinical studies [43], and there is a high correlation between IR estimations using HOMA-IR and the euglycemic clamp method [14]. An advantage of HOMA-IR compared to OGTT is that it can be performed in the initial acute phase of IS, and in severe strokes with for instance unconsciousness or dysphagia, situations in which OGTT may be detrimental and thus is contraindicated. Treatment of diabetes, e.g. insulin, could interfere with HOMA-IR calculations, but we excluded all diabetic patients and controls. However, we do not have full record of the on occasion medicine, and we therefore cannot rule out that a few patients could have received insulin injections in the acute phase of IS. Also, we do not have record of all other medications that could affect glucose homeostasis, e.g. thiazides. Another limitation is that blood samples usually were not taken directly after falling ill in IS; they were taken at 1-10 days (median 4 days), hence to a large extent reflecting stress hyperglycemia. However, if CRP, which more than NIHSS may reflect the acute stress response, was added to the binary logistic regression model, this did not change the associations exhibited in Table 3 (data not shown).

\section{Conclusions}

Our study shows that HOMA-IR is elevated both in the acute phase of IS and at the follow-up after 3 months, and that HOMA-IR associates with worse stroke severity in non-diabetic IS patients. Acute HOMA-IR, but not HOMA-IR at 3 months, was associated with worse functional outcome after 3 months and 7 years, but these associations became weaker and non-significant after adjustment for stroke severity. Possibly, this could mean that elevated HOMA-IR is part of the acute morbidity in IS and that the prognostic value of acute HOMA-IR is primarily linked to that of other negative prognostic factors including initial stroke severity. Finally, in the largest IS subtype cryptogenic stroke, we observed an association between acute HOMA-IR and poor outcome after 2 years that remained significant after adjustment for age and stroke severity. This indicates the IR may be a relatively more important factor in cryptogenic stroke than in other stroke subtypes, but the physiological relevance of this association needs to be explored in further studies.

\section{Abbreviations \\ Cl: 95\% confidence interval; HOMA-IR: The Homeostasis model assessment of IR; IR: Insulin resistance; IS: Ischemic stroke; mRS: modified Rankin Scale; NIHSS: National Institutes of Health Stroke Scale; SSS: Scandinavian Stroke Scale; TOAST: Trial of Org 10172 in Acute Stroke Treatment}

\section{Acknowledgements}

The authors thank research nurse Ingrid Eriksson for her excellent work and assistance with the study patients and controls. The authors also thank Dr. Per Ladenvall, MD, PhD and MSc Cecilia Lundholm for analyzing insulin levels.

\section{Authors' contributions}

DÅ planned, designed, analyzed, and wrote the paper. NDÅ planned and wrote the paper. KJ planned and wrote the paper. LHN planned and wrote the paper. PR planned and wrote the paper. CB planned and wrote the paper. Jl planned and wrote the paper. CJ planned, designed and wrote the paper. JS planned, analyzed and wrote the paper. All authors contributed to and have approved the final manuscript.

\section{Funding}

This study was supported by the Swedish Medical Society (Svenska Läkaresällskapet), grants from the Swedish Government (ALFGBG-438631) the Swedish Research Council (K2011-65X-14605-09-6), the Swedish Heart Lung Foundation (20100256), the Swedish Stroke Association, the Göteborg Foundation for Neurological Research, and the Yngve Land, Rune and Ulla Amlöv, Edit Jacobson, Magnus Bergvall, Emelle, Lars Hierta, and John and Brit Wennerström foundations.

\section{Availability of data and materials}

The datasets used and/or analyzed during the current study are available from the corresponding author upon reasonable request.

\section{Ethics approval and consent to participate}

This study was conducted in accordance with the 1964 Helsinki Declaration and its later amendments or comparable ethical standards. Participants or next-of-kin provided written informed consent. This study was approved by the Ethics Committee of the University of Gothenburg. The Ethics Committee of the University of Gothenburg is responsible for ethical applications from the entire region of western Sweden, covering all four hospitals in the study. 


\section{Competing interests}

The authors declare that they have no competing interests.

\begin{abstract}
Author details
'Department of Internal Medicine, Institute of Medicine, the Sahlgrenska Academy at University of Gothenburg, Gothenburg, Sweden. ${ }^{2}$ Center of Brain Repair and Rehabilitation, Institute of Neuroscience and Physiology, the Sahlgrenska Academy at University of Gothenburg, Gothenburg, Sweden. ${ }^{3}$ Department of Clinical Neuroscience, Institute of Neuroscience and Physiology, the Sahlgrenska Academy at University of Gothenburg, Gothenburg, Sweden. ${ }^{4}$ Department of Pathology and Clinical Genetics, Institute of Biomedicine, the Sahlgrenska Academy at University of Gothenburg, Gothenburg, Sweden. ${ }^{5}$ Department of Internal Medicine, Sahlgrenska University Hospital, University of Gothenburg, Blå stråket 5, SE-413 45 Göteborg, Sweden.
\end{abstract}

\section{Received: 28 September 2018 Accepted: 19 July 2019} Published online: 25 July 2019

\section{References}

1. Luitse MJ, Biessels GJ, Rutten GE, Kappelle $\amalg$. Diabetes, hyperglycaemia, and acute ischaemic stroke. The Lancet Neurology. 2012;11(3):261-71.

2. Kernan WN, Inzucchi SE, Viscoli CM, Brass LM, Bravata DM, Horwitz RI. Insulin resistance and risk for stroke. Neurology. 2002;59(6):809-15.

3. Rundek T, Gardener H, Xu Q, Goldberg RB, Wright CB, Boden-Albala B, Disla $N$, Paik MC, Elkind MS, Sacco RL. Insulin resistance and risk of ischemic stroke among nondiabetic individuals from the northern Manhattan study. Arch Neurol. 2010;67(10):1195-200.

4. Losser MR, Damoisel C, Payen D. Bench-to-bedside review: glucose and stress conditions in the intensive care unit. Crit Care. 2010;14(4):231.

5. Capes SE, Hunt D, Malmberg K, Pathak P, Gerstein HC. Stress hyperglycemia and prognosis of stroke in nondiabetic and diabetic patients: a systematic overview. Stroke. 2001;32(10):2426-32.

6. Megherbi SE, Milan C, Minier D, Couvreur G, Osseby GV, Tilling K, Di Carlo A, Inzitari D, Wolfe CD, Moreau T, et al. Association between diabetes and stroke subtype on survival and functional outcome 3 months after stroke: data from the European BIOMED stroke project. Stroke. 2003;34(3):688-94.

7. Bruno A, Biller J, Adams HP Jr, Clarke WR, Woolson RF, Williams LS, Hansen MD. Acute blood glucose level and outcome from ischemic stroke. Trial of ORG 10172 in acute stroke treatment (TOAST) investigators. Neurology. 1999:52(2):280-4.

8. Weir CJ, Murray GD, Dyker AG, Lees KR. Is hyperglycaemia an independent predictor of poor outcome after acute stroke? Results of a long-term follow up study. BMJ. 1997;314(7090):1303-6.

9. Tanaka R, Ueno Y, Miyamoto N, Yamashiro K, Tanaka Y, Shimura H, Hattori $\mathrm{N}$, Urabe T. Impact of diabetes and prediabetes on the short-term prognosis in patients with acute ischemic stroke. J Neurol Sci. 2013;332(1-2):45-50.

10. Lawlor DA, Fraser A, Ebrahim S, Smith GD. Independent associations of fasting insulin, glucose, and glycated haemoglobin with stroke and coronary heart disease in older women. PLoS Med. 2007;4(8):e263.

11. Li S, Yin C, Zhao W, Zhu H, Xu D, Xu Q, Jiao Y, Wang X, Qiao H. Homeostasis model assessment of insulin resistance in relation to the poor functional outcomes in nondiabetic patients with ischemic stroke. Biosci Rep. 2018;38(3)BSR20180330.

12. Jing J, Pan Y, Zhao X, Zheng H, Jia Q, Mi D, Chen W, Li H, Liu L, Wang C, et al. Insulin resistance and prognosis of nondiabetic patients with ischemic stroke: the ACROSS-China study (abnormal glucose regulation in patients with acute stroke across China). Stroke. 2017;48(4):887-93.

13. Ago T, Matsuo R, Hata J, Wakisaka Y, Kuroda J, Kitazono T, Kamouchi M. Insulin resistance and clinical outcomes after acute ischemic stroke. Neurology. 2018;90(17):e1470-7.

14. Matthews DR, Hosker JP, Rudenski AS, Naylor BA, Treacher DF, Turner RC. Homeostasis model assessment: insulin resistance and beta-cell function from fasting plasma glucose and insulin concentrations in man. Diabetologia. 1985;28(7):412-9.

15. Åberg D, Jood K, Blomstrand C, Jern C, Nilsson M, Isgaard J, Åberg ND. Serum IGF-I levels correlate to improvement of functional outcome after ischemic stroke. J Clin Endocrinol Metab. 2011;96(7):E1055-64.

16. Jood K, Ladenvall C, Rosengren A, Blomstrand C, Jern C. Family history in ischemic stroke before 70 years of age: the Sahlgrenska Academy study on ischemic stroke. Stroke. 2005;36(7):1383-7.
17. Adams HP Jr, Bendixen BH, Kappelle LJ, Biller J, Love BB, Gordon DL, Marsh EE 3rd. Classification of subtype of acute ischemic stroke. Definitions for use in a multicenter clinical trial. TOAST. Trial of org 10172 in acute stroke treatment. Stroke. 1993;24(1):35-41.

18. Olsson S, Holmegaard L, Jood K, Sjogren M, Engstrom G, Lovkvist H, Blomstrand C, Norrving B, Melander O, Lindgren A, et al. Genetic variation within the interleukin-1 gene cluster and ischemic stroke. Stroke. 2012;43(9): 2278-82.

19. Ali K, Cheek E, Sills S, Crome P, Roffe C. Development of a conversion factor to facilitate comparison of National Institute of health stroke scale scores with Scandinavian stroke scale scores. Cerebrovasc Dis. 2007;24(6):509-15.

20. Gray $\sqcup$, Ali M, Lyden PD, Bath PM. Virtual international stroke trials archive C: interconversion of the National Institutes of Health stroke scale and Scandinavian stroke scale in acute stroke. J Stroke Cerebrovascular Dis. 2009; 18(6):466-8.

21. JR Gavinlll (chairman) et al. Report of the Expert Committee on the Diagnosis and Classification of Diabetes Mellitus. Diabetes Care. 1997;20(7):1183-7.

22. Giles TD, Berk BC, Black HR, Cohn JN, Kostis JB, Izzo JL Jr, Weber MA. Expanding the definition and classification of hypertension. J Clin Hypertens. 2005;7(9):505-12.

23. Bang OY, Ovbiagele B, Kim JS. Nontraditional risk factors for ischemic stroke: an update. Stroke. 2015;46(12):3571-8.

24. Åberg ND, Olsson S, Åberg D, Jood K, Stanne TM, Nilsson M, Blomstrand C, Svensson J, Isgaard J, Jern C. Genetic variation at the IGF1 locus shows association with post-stroke outcome and to circulating IGF1. Eur J Endocrinol. 2013;169(6):759-65.

25. Persson J, Holmegaard L, Karlberg I, Redfors P, Jood K, Jern C, Blomstrand C, Forsberg-Warleby G. Spouses of stroke survivors report reduced healthrelated quality of life even in long-term follow-up: results from Sahlgrenska Academy study on ischemic stroke. Stroke. 2015;46(9):2584-90.

26. Scott JF, Robinson GM, French JM, O'Connell JE, Alberti KG, Gray CS. Prevalence of admission hyperglycaemia across clinical subtypes of acute stroke. Lancet. 1999;353(9150):376-7.

27. Calleja Al, Garcia-Bermejo P, Cortijo E, Bustamante R, Rojo Martinez E, Gonzalez Sarmiento E, Fernandez-Herranz R, Arenillas JF. Insulin resistance is associated with a poor response to intravenous thrombolysis in acute ischemic stroke. Diabetes Care. 2011;34(11):2413-7.

28. Bas DF, Ozdemir AO, Colak E, Kebapci N. Higher insulin resistance level is associated with worse clinical response in acute ischemic stroke patients treated with intravenous thrombolysis. Transl Stroke Res. 2016;7(3):167-71.

29. Uyttenboogaart M, Koch MW, Stewart RE, Vroomen PC, Luijckx GJ, De Keyser J. Moderate hyperglycaemia is associated with favourable outcome in acute lacunar stroke. Brain. 2007;130(Pt 6):1626-30.

30. Fang $Y$, Zhang S, Wu B, Liu M. Hyperglycaemia in acute lacunar stroke: a Chinese hospital-based study. Diab Vasc Dis Res. 2013;10(3):216-21.

31. Williams LS, Rotich J, Qi R, Fineberg N, Espay A, Bruno A, Fineberg SE, Tierney WR. Effects of admission hyperglycemia on mortality and costs in acute ischemic stroke. Neurology. 2002;59(1):67-71.

32. Baird TA, Parsons MW, Phan T, Butcher KS, Desmond PM, Tress BM, Colman $P G$, Chambers BR, Davis SM. Persistent poststroke hyperglycemia is independently associated with infarct expansion and worse clinical outcome. Stroke. 2003;34(9):2208-14.

33. Ginsberg MD. Hyperglycemia and stroke outcome: vindication of the ischemic penumbra. Ann Neurol. 2002;52(1):5-6.

34. Boden-Albala B, Sacco RL, Lee HS, Grahame-Clarke C, Rundek T, Elkind MV Wright C, Giardina EG, DiTullio MR, Homma S, et al. Metabolic syndrome and ischemic stroke risk: northern Manhattan study. Stroke. 2008;39(1):30-5.

35. Kodama K, Tojjar D, Yamada S, Toda K, Patel CJ, Butte AJ. Ethnic differences in the relationship between insulin sensitivity and insulin response: a systematic review and meta-analysis. Diabetes Care. 2013;36(6):1789-96.

36. Ahmed N, Davalos A, Eriksson N, Ford GA, Glahn J, Hennerici M, Mikulik R, Kaste M, Lees KR, Lindsberg PJ, et al. Association of admission blood glucose and outcome in patients treated with intravenous thrombolysis: results from the safe implementation of treatments in stroke international stroke thrombolysis register (SITS-ISTR). Arch Neurol. 2010;67(9):1123-30.

37. Robinson LE, van Soeren $\mathrm{MH}$. Insulin resistance and hyperglycemia in critical illness: role of insulin in glycemic control. AACN Clin Issues. 2004;15(1):45-62.

38. Farooqui AA, Farooqui T, Panza F, Frisardi V. Metabolic syndrome as a risk factor for neurological disorders. Cel Mol Life Sci. 2012;69(5):741-62.

39. Laou E, Milionis H, Petrou A, Arnaoutoglou E, Glantzounis G, Bairaktari E, Mavridis D, Mikhailidis DP, Papadopoulos G, Tzimas P. The impact of 
metabolic syndrome and its components on perioperative outcomes after elective laparotomy - a prospective observational study. Am J Surg. 2017; 214(5):831-7.

40. Kernan WN, Viscoli CM, Furie KL, Young LH, Inzucchi SE, Gorman M, Guarino PD, Lovejoy AM, Peduzzi PN, Conwit R, et al. Pioglitazone after ischemic stroke or transient ischemic attack. N Engl J Med. 2016;374(14):1321-31.

41. Schulz UG, Rothwell PM. Differences in vascular risk factors between etiological subtypes of ischemic stroke: importance of population-based studies. Stroke. 2003;34(8):2050-9.

42. Fonseca AC, Ferro JM. Cryptogenic stroke. Eur J Neurol. 2015;22(4):618-23.

43. Wallace TM, Levy JC, Matthews DR. Use and abuse of HOMA modeling. Diabetes Care. 2004;27(6):1487-95.

\section{Publisher's Note}

Springer Nature remains neutral with regard to jurisdictional claims in published maps and institutional affiliations.

Ready to submit your research? Choose BMC and benefit from:

- fast, convenient online submission

- thorough peer review by experienced researchers in your field

- rapid publication on acceptance

- support for research data, including large and complex data types

- gold Open Access which fosters wider collaboration and increased citations

- maximum visibility for your research: over $100 \mathrm{M}$ website views per year

At $\mathrm{BMC}$, research is always in progress.

Learn more biomedcentral.com/submissions 